\title{
Genomic characteristics of a chicken infectious anemia virus strain isolated from contaminated chicken pox attenuated vaccine
}

\section{Longfei Chen}

Shandong Agricultural University

\section{Yan Li}

Shandong Agricultural University

Jinjin Wang

Shandong Agricultural University

Yawen Zhang

Shandong Agricultural University

Shuang Chang

Shandong Agricultural University

\section{Yixin Wang}

Shandong Agricultural University

Peng Zhao ( $\nabla$ zhaopeng@sdau.edu.cn )

Shandong Agricultural University

\section{Research Article}

Keywords: Chicken infectious anemia virus, Recombinase-aided amplification Assay, contamination, genome analysis, live vaccines

Posted Date: October 4th, 2021

DOI: https://doi.org/10.21203/rs.3.rs-954966/v1

License: (c) (1) This work is licensed under a Creative Commons Attribution 4.0 International License. Read Full License 


\section{Abstract}

Chicken infectious anemia virus (CIAV) can be transmitted by contaminated live vaccines, and causes huge economic losses. This study evaluated the contamination status of CIAV in 24 batches of vaccines by recombinase-aided amplification assay (RAA), fluorescence quantitative PCR and dot blot assay, and then determined a contaminated avian pox attenuated vaccine. The whole genome of the CIAV contaminant was then sequenced and named as JS2020-PFV (Genbank accession number: MW234428, 2296bp). It showed 94.5-99.9\% identities with reference strains, and shared the closest evolution relationship with $A B 1 \mathrm{~K}$ which was isolated from a chicken farm in Turkey. All these suggested that the use of CIAV contaminated live vaccine may be the reason for its epidemic in poultry.

\section{Introduction}

Chicken infectious anemis virus (CIAV) induces severe anemia and immunosuppression in poultry (Rosenberger et al.,1998). After its first report in 1979 (Yuasa et al., 1979), CIAV has been detected worldwide and has caused huge economic losses (Fatoba et al.,2019). Recently, CIAV infections have also frequently appeared in different chicken farms in China (Zhang et al., 2013; Fang et al., 2017; Li et al., 2017; Su et al., 2019; Tan et al., 2020). The pathogenesis of CIAV has been well understood that it mainly causes the atrophy of bone marrow, hematopoietic tissue, and lymphatic tissues (e.g., thymus) in young chickens (Adair et al.,2000). CIAV can be transmitted both vertically and horizontally via respiratory and digestive tracts (ref), but how it spreads across different regions and even countries remains unclear.

It is worth noting that the first strain of CIAV was isolated from the contaminated attenuated vaccine (Yuasa et al., 1979). And, after that, similar reports emerged in a lot of countries (Marin et al., 2013; Varela et al., 2014; Li et al., 2017), revealing that the use of contaminated live vaccines is an important way for the transmission of CIAV. Through interactions with attenuated vaccine strains or co-infected viruses, CIAV triggers more serious clinical symptoms with strong immunosuppressive ability (Su et al., 2018 a,b; Su et al., 2019). More importantly, the attenuated vaccines are usually inoculated at a low age, the CIAV infection caused by it will greatly interfere with the immune responses against vaccines, resulting in secondary infection (Zhang et al., 2017; Su et al., 2019). Therefore, monitoring the vaccine contaminations is of great significance for preventing CIAV infection in poultry.

As a relatively conservative DNA virus, the detection of CIAV is relatively easy, and there are a large number of published methods (Han et al., 2019; Techera et al., 2019; Li et al., 2019). Although the sensitivity and accuracy of these methods are very high, they are not suitable for farms to detect the contamination in vaccines due to long operation time, complex experiments or high price. Recombinaseaided amplification (RAA) is a new isothermal amplification technology, requiring no classical thermostable enzyme. RAA has been widely used to detect multiple viruses, even the SARS-CoV-19 (Wu et al.,2020). The reaction, containing a recombinase UvsX obtained from Escherichia coli and the single strand DNA-binding protein (SSB) used in a polymerase chain reaction (PCR), can form single-stranded 
DNA without heating and can be finished within 20 min at a constant temperature of $39^{\circ} \mathrm{C}($ Rohrman et al.,2012).

This study aims to use RAA to moniter the CIAV contamination status of live vaccines, and verify its efficacy by comparing to fluorescence quantitative PCR assay (qPCR) and dot blot. The whole genome of contaminated CIAV strain was sequenced for further analysis.

\section{Materials And Methods}

\section{Vaccine collection}

24 batchs of live vaccines against Newcastle disease virus, infectious bronchitis, infectious laryngotracheitis encephalomyelitis, avian pox, Marek disease virus, mycoplasma gallisepticum, viral arthritis, were collected from chicken farms. All these vaccines were diluted with normal saline according to the instructions, and $200 \mu \mathrm{l}$ of the dilute was used for DNA extraction.

\section{DNA Extraction}

DNA was extracted using the DNA extraction kit (Omega, Bio-Tek, USA) according to the manufacturer's instructions as the template for RAA, qPCR and dot blot.

\section{RAA}

The primers and probe were designed according to the published CIAV genome (Table 1; Table2). The RAA fluorescence kit and RAA amplification instrument were provided by Hangzhou ZC Bio-Sci \& Tech Co., Ltd (Hangzhou, China). dd $\mathrm{H}_{2} \mathrm{O}$ was used as the negative control and standard plasmid contianing full-length CIAV genomes was contructed by our lab and used as positive control(Li et al. 2019). RAA detection methods and primer probes are shown in Table 1. 
Table 1

Sequence of primers used in this study

\begin{tabular}{|c|c|c|}
\hline Primers & Sequence & $\begin{array}{l}\text { Product } \\
\text { length }\end{array}$ \\
\hline C-F & CAGTAGGTATACGCAAGGCGGTCCGGGTG & $277 b p$ \\
\hline C-R & CACACAGCGATAGAGTGATTGTAATTCCAG & \\
\hline C-probe & $\begin{array}{l}\text { CAAGTAATTTCAAATGAACGCTCTCCAAGA[FAM-dT]A[THF][BHQ1- } \\
\text { dT]CCACCCGGACCATCAAC }\end{array}$ & \\
\hline $\begin{array}{l}\text { CAV- } \\
\text { com-F1 }\end{array}$ & GCATTCCGAGTGGTTACTATTCC & $842 b p$ \\
\hline $\begin{array}{l}\text { CAV- } \\
\text { com-R1 }\end{array}$ & CGTCTTGCCATCTTACAGTCTTA & \\
\hline $\begin{array}{l}\text { CAV- } \\
\text { com-F2 }\end{array}$ & CGAGTACAGGGTAAGCGAGCTAAA & $990 b p$ \\
\hline $\begin{array}{l}\text { CAV- } \\
\text { com-R2 }\end{array}$ & TGCTATTCATGCAGCGGACTT & \\
\hline $\begin{array}{l}\text { CAV- } \\
\text { com-F3 }\end{array}$ & ACGAGCAACAGTACCCTGCTA & $802 b p$ \\
\hline $\begin{array}{l}\text { CAV- } \\
\text { com-R3 }\end{array}$ & CTGTACATGCTCCACTCGTT & \\
\hline
\end{tabular}


Table 2

CIAV strains were used as reference strains in this study

\begin{tabular}{|c|c|c|c|c|}
\hline Strains & Country and Time & Host & Accession NO. & Whole length \\
\hline SDLY08 & 2008 China & Chicken & FJ172347 & 2298 bp \\
\hline 3711 & 2007 Australia & Chicken & EF683159 & 2279 bp \\
\hline CA V-6 & 2014 China & Chicken & KJ728817 & 2298 bp \\
\hline CA V-14 & 2014 China & Chicken & KJ728824 & 2298 bp \\
\hline E51057 & 2000 Japan & Chicken & E51057 & 2298 bp \\
\hline SC-MZ & 2014 China & Chicken & KM496306 & 2298 bp \\
\hline CAT-CA V & 2014 China & Chicken & KC414026 & 2295 bp \\
\hline CIA V89-69 & 2013 Korea & Chicken & JF507715 & 2298 bp \\
\hline GD-1-12 & 2012 China & Chicken & JX260426 & 2298 bp \\
\hline LF4 & 2005 China & Chicken & AY839944 & 2298 bp \\
\hline HH982173 & 2006 USA & Chicken & $\mathrm{HH} 982173$ & 2298 bp \\
\hline AB031296 & 2000 Japan & Chicken & AB031296 & 2298 bp \\
\hline AGV2 & 2012 China & Human & JQ690762 & 2316 bp \\
\hline L14767 & 1999 USA & Chicken & L14767 & 2298 bp \\
\hline $3-1$ & 2003 Malaysia & Chicken & AF390038 & 2298 bp \\
\hline A48606 & 1996 USA & Chicken & A48606 & $2298 \mathrm{bp}$ \\
\hline CAU66304 & 1997 UK & Chicken & U66304 & 2319 bp \\
\hline clone 33 & 2002 Germany & Chicken & AJ297684 & 2298 bp \\
\hline NC001427 & 2015 USA & Chicken & NC001427 & 2319 bp \\
\hline Cux-1 & 2008 Netherlands & Chicken & M55918 & 2319 bp \\
\hline DI072479 & 1990 USA & Chicken & DI072479 & 2298 bp \\
\hline CAV-4 & 2014 China & Chicken & KJ728816 & 2298 bp \\
\hline CAV-18 & 2014 China & Chicken & KJ728827 & 2298 bp \\
\hline GD-K-12 & 2013 China & Chicken & KF224935 & 2298 bp \\
\hline $\mathrm{AH} 4$ & 2005 China & Chicken & DQ124936 & 2298 bp \\
\hline AB119448 & 2009 Japan & Chicken & AB119448 & 2298 bp \\
\hline TR20 & 1999 Japan & Chicken & AB027470 & $2298 \mathrm{bp}$ \\
\hline
\end{tabular}




\begin{tabular}{|c|c|c|c|c|}
\hline Strains & Country and Time & Host & Accession NO. & Whole length \\
\hline Cuxhaven & 1992 Germany & Chicken & M81223 & 2298 bp \\
\hline AB1K & 2020 Turkey & Chicken & MT259319 & 2296 bp \\
\hline M81223 & 1993 Germany & Chicken & M81223 & 2298 bp \\
\hline CAU65414 & 1996 Australia & Chicken & CAU65414 & 2298 bp \\
\hline A2 & 2000 Japan & Chicken & AB031296 & 2298 bp \\
\hline AF313470 & 2000 USA & Chicken & AF313470 & 2294 bp \\
\hline AF227982 & 2001 Australia & Chicken & AF227982 & 2286 bp \\
\hline AB046590 & 2001 Japan & Chicken & AB046590 & 2298 bp \\
\hline AF475908 & 2002 China & Chicken & AF475908 & 2298 bp \\
\hline clone 34 & 2002 Germany & Chicken & AJ297685 & 2297 bp \\
\hline SMSC-1P60 & 2003 Malaysia & Chicken & AF390102 & 2298 bp \\
\hline SMSC-1 & 2003 Malaysia & Chicken & AF285882 & 2298 bp \\
\hline BD-3 & 2004 Bangladesh & Chicken & AF395114 & 2298 bp \\
\hline $\mathrm{C} 14$ & 2004 China & Chicken & EF176599 & 2298 bp \\
\hline SD22 & 2005 China & Chicken & DQ141673 & 2298 bp \\
\hline DQ217401 & 2005 Malaysia & Chicken & SMSC-1P123 & 2298 bp \\
\hline CAE26P4 & 2007 Netherlands & Chicken & D10068 & 2298 bp \\
\hline $01-4201$ & 2007 USA & Chicken & DQ991394 & 2298 bp \\
\hline Cuxhaven-1 & 2008 Netherlands & Chicken & M55918 & 2319 bp \\
\hline CAECA123 & 2008 Japan & Chicken & D31965 & $2319 b p$ \\
\hline 98D02152 & 2010 USA & Chicken & AF311892 & 2298 bp \\
\hline GXC060821 & 2012 China & Chicken & JX964755 & 2292 bp \\
\hline CAV-10 & 2014 Argentina & Chicken & KJ872513 & 2298 bp \\
\hline KM496307 & 2014 China & Chicken & SC-MZ42A & 2298 bp \\
\hline Isolate 18 & 2014 Taiwan & Chicken & KJ728827 & 2298 bp \\
\hline SD15 & 2015 China & Chicken & KX811526 & 2298 bp \\
\hline JS2020-PFV & 2020 China & Chicken & MW234428 & 2296 bp \\
\hline GX1804 & 2018 China & Chicken & MK484615 & 2298 bp \\
\hline
\end{tabular}




\begin{tabular}{|lllll|}
\hline Strains & Country and Time & Host & Accession NO. & Whole length \\
\hline SD1510 & 2016 China & Chicken & KU598851 & 2298 bp \\
HN1405 & 2016 China & Chicken & KU645520 & 2298 bp \\
\hline
\end{tabular}

Table 3

Position of mutational VP1 amino acid of JS2020-PFV

\begin{tabular}{|c|c|c|c|c|c|c|c|c|c|c|c|}
\hline \multirow[t]{2}{*}{ Strains } & \multicolumn{11}{|c|}{ Position of VP1 amino acid } \\
\hline & 89 & 92 & 125 & 139 & 141 & 144 & 157 & 254 & 370 & 447 & 448 \\
\hline Cux-1 & $\mathrm{T}$ & G & 1 & K & Q & D & V & G & $S$ & $\mathrm{~T}$ & Q \\
\hline GD-F-1 & $\mathrm{T}$ & G & L & K & Q & $\mathrm{E}$ & M & $\mathrm{E}$ & G & $S$ & Q \\
\hline SDLY08 & $\mathrm{T}$ & G & 1 & K & $\mathrm{R}$ & E & V & E & G & $\mathrm{T}$ & Q \\
\hline SD15 & $\mathrm{T}$ & G & 1 & K & Q & Q & V & $E$ & $\mathrm{~T}$ & $S$ & $\mathrm{P}$ \\
\hline AB1K & $\mathrm{T}$ & $\mathrm{D}$ & 1 & K & Q & E & M & G & $S$ & $\mathrm{~T}$ & $\mathrm{Q}$ \\
\hline JS2020-PFV & $\mathrm{K}$ & D & I & K & Q & E & $M$ & $G$ & $S$ & $\mathrm{~T}$ & $\mathrm{H}$ \\
\hline
\end{tabular}

\section{Sample detection with fluorescent quantitative PCR}

With the fluorescent quantitative PCR kit produced by TaKaRa Biotechnology (Takara, Dalian, Chian), the sample DNA was detected and verified by the fluorescent quantitative PCR method according to the references (Li et al. 2019).

\section{Sample detection with dot blot hybridization}

The samples were tested by PCR combined with dot blot hybridization (Meng et al. 2018), and the nitrocellulose membrane was purchased from Boehringer Millipore (Merck, Germany).

\section{Amplification and sequencing of whole genome of samples}

According to the sequence published in the GenBank, three primers were designed to amplify the sample DNA using DNAStar 6.0 application. The primers are shown in Table 1. The amplification procedure was as follows: $94^{\circ} \mathrm{C}$ for $5 \mathrm{~min} ; 94^{\circ} \mathrm{C}$ for $30 \mathrm{~s}, 55^{\circ} \mathrm{C}$ for $30 \mathrm{~s}$, and $72^{\circ} \mathrm{C}$ for $30 \mathrm{~s}$ (34 cycles); $72^{\circ} \mathrm{C}$ for 10 min (35 cycles); and $4^{\circ} \mathrm{C}$. The PCR products were identified using $1 \%$ agarose gel electrophoresis and stained with ethidium bromide. The bands were purified with Omega gel recovery and purification kit (Omega BioTek, Norcross, GA, USA), and the recovered target fragments were attached to the pMD18-T vector (Takara, Dalian, Chian). After overnight ligation at $16^{\circ} \mathrm{C}$, the ligation products were transformed into competent cells of E. coli DH5a(Takara, Japan), and the positive clones were screened. Finally, the bacterial liquid identified as positive by PCR was sent to Shanghai Bioengineering Co., Ltd. for sequencing.

\section{Sequence analysis}


The DNA sequences were assembled using DNAStar (version 6.0). Multiple sequence alignment was performed using the Clustal W (BioEdit version 7.0) program, and the comparison of sequence identity was performed using MegAlign software (DNAStar). Phylogenetic analysis was performed using the maximum likeihood (ML) method on RAxML.

\section{Results}

\section{Results of CIAV nucleic acid test in vaccines}

RAA, qPCR assay, and dot blot were employed to detect CIAV contamination in live vaccines. Results showed that an avian pox attenuated vaccine sample were determined as CIAV positive in all three methods (Figure 1). These results also suggested that the sensitivity and accuracy of RAA is similar to that of qPCR and dot blot, hence it can be used in daily moniter of vaccine contamination.

\section{Whole genome sequencing}

In order to further reveal the molecular characteristics of the CIAV contaminants, the whole genome of this strain was sequenced using three pairs of primers. Electrophoretic analysis confirmed the positive amplication by PCR, and the three segments were $842 \mathrm{bp}, 990 \mathrm{bp}$ and $802 \mathrm{bp}$, respectively (Figure 2), which was consistent with the expected band size. Furthermore, the whole genome of this isolate was $2296 \mathrm{bp}$, covering three open-reading-frame (ORF), namely VP1, VP2, and VP3.

The lengths of VP1, VP2 and VP3 were $1350 \mathrm{bp}, 651 \mathrm{bp}$ and $366 \mathrm{bp}$, respectively. The isolate was named JS2020-PFV and its sequence was uploaded to GenBank gene library with the accession number of MW234428.

\section{Genome analysis of JS2020-PFV}

The whole genome of JS2020-PFV strain was compared with that of 56 other CIAV strains. Results showed that JS2020-PFV shared 94.5-99.9\% identities with other reference strains. Among them, JS2020-PFV had the highest identity (99\%) with the AB1K strain (MT259319) isolated from a chicken farm in Turkey. A phylogenetic tree was constructed based on the whole genome sequence of JS2020PFV and reference strains, and the results further confirmed the closest evolution relationship between JS2020-PFV and AB1K (Figure 3).

\section{Analysis of VP1 gene variation in JS2020-PFV strain}

The VP1 gene of JS2020-PFV strain shared 94.0-99.9\% identities with the reference strains, with the highest identity (99.9\%) with AB1K. The genetic evolution relationship between the VP1 gene of JS2020PFV strain and reference strains was consistent with the whole genome sequence (Figure 3). Further analysis of the VP1 gene of JS2020-PFV strain revealed that there are two amino acid mutations within which had never been found before, namely threonine at position 89 of VP1 protein changed to lysine and glutamine at position 448 changed to lysine (Table 2). 


\section{Difference analysis of transcriptional regulatory elements in non-coding regions}

Clustal W method was used for multiple alignment analysis of the non-coding regions of JS2020-PFV with 9 reference strains. Compared with the reference strains, the the motif in non-coding regions of CIAV was highly conserved (Figure 4). Softberry's Nsite online service analysis showed that four CREB sites associated with viral apoptotic capacity were also conserved in the new isolate. The JS2020-PFV and most of the reference strains all had four DR regions in the non-coding region, except for one additional DR region in the CUX-1 strain.

\section{Discussion}

Over the past two decades, many live vaccines have been proved to be contaminated with exogenous viruses, including avian leukosis virus (Mao et al., 2020), fowl adenovirus (Su et al., 2020), reticuloendotheliosis virus ( $\mathrm{Li}$ et al., 2015), CIAV (Li et al., 2017) and others. This phenomenon is mainly caused by the use of SPF chicken embryos infected with exogenous viruses (Li et al., 2016). The use of contaminated vaccines will not only cause the spread of the virus, but also cause serious clinical symptoms, especially vaccines contaminated with CIAV that induces strong immunosuppression. Previsous studies demonstrated the the co-infection with CIAV can significantly improve the pathogenicity of the LaSota strain in NDV vaccines, and decrease the antibody titer, which makes the vaccinated chickens vulnerable to wild NDV strains (Su et al., 2019). Therefore, monitering the exogenous viruses contamination in live vaccination is of great significance for poultry farms.

Currently, the standard routine for detecting exogenous virus contamination in vaccines mainly depend on cell culture and SPF chicken inspections, but the working cycle of the above methods is too long, so it is not conducive to the vaccine work in farms. Alternatively, several molecular detection methods were established for detecting exogenous viruses, such as CIAV and FAdV (Li et al., 2019; Dong et al., 2019). However, none of them can be directly used in farms because of complex equipment or difficult operations.

In this study, RAA assay, which can be finished with 15 minutes in a hand-held device, was employed to detect the contaminated CIAV in live vaccines. We designed the primers and probe based on the published CIAV genomes, and optimized the methods using the constructed plasmid containing the whole genome of CIAV ( $\mathrm{Li}$ et al. 2019). After that, this method was used to analyze 24 batchs of live vaccine, and the traditional qPCR and dot blot were used at the same time to verify the results of RAA tests. Results found that one avain pox vaccine was positive for CIAV in all these three methods indicating that the detection results were true and reliable. It should be noted that RAA completed the whole detection process in only 15 minutes, while qPCR and dot blot took 1.5 and 14 hours respectively. Therefore, the RAA method is more suitable for monitoring vaccine contamination in farms because of its flexibility and convenience. 
Furthermore, the whole genome sequence of CIAV was sequenced and named as JS2020-PFV. It was found that JS2020-PFV had extremely high identities (99\%) and the closest evolution relationship with the AB1K strain (MT259319) that isolated from a chicken farm in Turkey. Besides, JS2020-PFV shared the same batch with Dutch isolate (D10068), American isolate (DI072479), American isolate (L14767) and Bangladesh isolate BD-3 (AF395114), while it was relatively unrelated to chinese isolates, including SDLY18 (FJ172347), GD-K-12 (KF224935) and SD15 (KX811526). These results are consistent with the fact that the vaccine was imported from a foreign company, suggesting that the transnational trade of vaccines may be an important way for some strains to spread across countries and regions.

The amino acid positions of VP1 protein are closely related to CIAV pathogenicity and cell proliferation (Fang et al. 2019). Previous studies have shown that amino acid positions 139 and 144 on VP1 were associated with the efficiency of virus proliferation and transmission in MSB1 cells (Yamaguchi et al. 2001). The amino acid positions 139 and 144 on the VP1 protein of JS2020-PFV are lysine (K) and glutamic acid $(E)$, which are consistent with the strain with high replication ability (Yamaguchi et al. 2001). Besides, glutamine (Q) at position 394 was associated with higher pathogenicity, while histidine (H) at position 394 was related with lower pathogenicity (Yamaguchi et al. 2001). In this study, the amino acid at position 394 of VP1 protein of JS2020-PFV is glutamine (Q), suggesting that this strain may also be highly pathogenic. There are more than ten motifs related to transcriptional regulation in the noncoding region of CAV, which are closely related to viral replication and transcriptional regulation. After comparing and analyzing the non-coding regions of the selected strains in this paper, it was found that these motifs were very conservative. We speculate that these related motifs may be necessary for the replication of the virus itself.

Overall, CIAV contamination was detected from a FPV vaccine by RAA, and genome analysis suggested that it may be a highly pathogenic strain, which reminded us to pay close attention to the possible contamination of exogenous virus in live poultry vaccine.

\section{Declarations}

\section{CONFLICT OF INTEREST STATEMENT}

The authors declare that the research was conducted in the absence of any commercial or financial relationships that could be construed as a potential conflict of interest.

\section{FUNDING INFORMATION}

This work was supported by the National Key Research and Development Program of China (2018YFD0500106).

\section{References}


Adair, B. M. 2000. Immunopathogenesis of chicken anemia virus infection. Dev. Comp. Immunol. 24:24755 .

Dong, G., F. Meng, Y. Zhang, Z. Cui, Lidan. H, S. Chang, and P. Zhao. 2019. Development and evaluation of a droplet digital PCR assay for the detection of fowl adenovirus serotypes 4 and 10 in attenuated vaccines. J. Virol. Methods. 265, 59-65.

Fang, L., Y. Li, Y. Wang, J. Fu, S. Cui, Li. X, S. Chang, and P. Zhao. 2017. Genetic Analysis of Two Chicken Infectious Anemia Virus Variants-Related Gyrovirus in Stray Mice and Dogs: The First Report in China, 2015. Biomed. Res. Int. 2017:6707868.

Fatoba, A. J., and A. Adeleke. 2019. Chicken anemia virus: A deadly pathogen of poultry. Acta. Virol. 63:19-25.

Han, C., X. Niu, L. Liu, J. Li, J. Li, S. Yao, X. Song, H. Gao, Y. Gao, X. Qi, X. Zeng, Y. Wang, and X. Wang. 2019. Development of a loop-mediated isothermal amplification assay for the detection of chicken anemia virus. Poult. Sci. 98:1176-1180.

Li, Q., Y. Zhang, F. Meng, H. Jiang, G. Xu, J. Ding, Y. Zhang, G. Dong, S. Tian, S. Chang, and P. Zhao. 2019. A New Strategy for the Detection of Chicken Infectious Anemia Virus Contamination in Attenuated Live Vaccine by Droplet Digital PCR. Biomed. Res. Int. 2019:2750472.

Li, Y., L. Fang, S. Cui, J. Fu, X. Li, H. Zhang, Z. Cui, S. Chang, W. Shi, and P. Zhao. 2017. Genomic C.haracterization of Recent Chicken Anemia Virus Isolates in China. Front. Microbiol. 8:401.

Li, Y., Y. Hu, S. Cui, J. Fu, Y. Wang, Z. Cui, L. Fang, S. Chang, and P. Zhao. 2017. Molecular characterization of chicken infectious anemia virus from contaminated live-virus vaccines. Poult. Sci. 96:1045-1051.

Li, Y., Y. Wang, L. Fang, J. Fu, S. Cui, Y. Zhao, Z. Cui, S. Chang, and P. Zhao. 2016. Genomic Analysis of the Chicken Infectious Anemia Virus in a Specific Pathogen-Free Chicken Population in China. Biomed. Res. Int. 2016:4275718.

Mao, Y., Q. Su, J. Li, T. Jiang, and Y. Wang. 2020. Avian leukosis virus contamination in live vaccines: A retrospective investigation in China. Vet. Microbiol. 246:108712.

Meng, F., G. Dong, Y. Zhang, S. Tian, Z. Cui, S. Chang, and P. Zhao. 2018. Co-infection of fowl adenovirus with different immunosuppressive viruses in a chicken flock. Poult. Sci. 97:1699-1705.

Rohrman, B. A., and R. R. Richards-Kortum. 2012. A paper and plastic device for performing recombinase polymerase amplification of HIV DNA. Lab. Chip. 12:3082-3088.

Rosenberger, J. K., and S. S. Cloud. 1998. Chicken anemia virus. Poult. Sci. 77:1190-2. 
Su, Q., L. Hou, Y. Gao, X. Liu, Z. Cui, S. Chang, and P. Zhao. 2020. Research Note: Molecular relationship of the fowl adenovirus serotype 4 isolated from the contaminated live vaccine and wild strains isolated in China, 2013-2018. Poult. Sci. 99:6643-6646.

Su, Q., Y. Li, F. Meng, Z. Cui, S. Chang, and P. Zhao. 2018. Newcastle disease virus-attenuated vaccine cocontaminated with fowl adenovirus and chicken infectious anemia virus results in inclusion body hepatitis-hydropericardium syndrome in poultry. Vet. Microbiol. 218:52-59.

Su, Q., Y. Li, Y. Zhang, Z. Zhang, F. Meng, Z. Cui, S. Chang, and P. Zhao. 2018. Newcastle disease virusattenuated vaccine LaSota played a key role in the pathogenicity of contaminated exogenous virus. Vet. Res. 49:80.

Su, Q., F. Meng, Y. Li, Y. Zhang, Z. Zhang, Z. Cui, S. Chang, P. Zhao. 2019. Chicken infectious anemia virus helps fowl adenovirus break the protection of maternal antibody and cause inclusion body hepatitishydropericardium syndrome in layers after using co-contaminated Newcastle disease virus-attenuated vaccine. Poult. Sci. 98:621-628.

Su, Q., T. Wang, F. Meng, Z. Cui, S. Chang, and P. Zhao. 2019. Synergetic pathogenicity of Newcastle disease vaccines LaSota strain and contaminated chicken infectious anemia virus. Poult. Sci. 98:19851992.

Su, Q., Y. Zhang, Y. Li, Z. Cui, S. Chang, and P. Zhao. 2019. Epidemiological investigation of the novel genotype avian hepatitis $E$ virus and co-infected immunosuppressive viruses in farms with hepatic rupture haemorrhage syndrome, recently emerged in China. Transbound. Emerg. Dis. 66:776-784.

Tan, C., Z. Wang, X. Lei, J. Lu, Z. Yan, J. Qin, F. Chen, Q. Xie, and W. Lin. 2020. Epidemiology, molecular characterization, and recombination analysis of chicken anemia virus in Guangdong province, China. Arch. Virol. 165:1409-1417.

Techera, C., G. Tomás, Y. Panzera, A. Banda, P. Perbolianachis, R. Pérez, and A. Marandino. 2019. Development of real-time PCR assays for single and simultaneous detection of infectious bursal disease virus and chicken anemia virus. Mol. Cell. Probes. 43:58-63.

Varela, A. P., H. F. Dos. Santos, S. P. Cibulski, C. M. Scheffer, C. Schmidt, F. E. Sales. Lima., A. D. Silva, P. A. Esteves, A. C. Franco, and P. M. Roehe. 2014. Chicken anemia virus and avian gyrovirus 2 as contaminants in poultry vaccines. Biologicals. 42:346-50.

Wu, T., Y. Ge, K. Zhao, X. Zhu, Y. Chen, B. Wu, F. Zhu, B. Zhu, and L. Cui. 2020. A reverse-transcription recombinase-aided amplification assay for the rapid detection of $\mathrm{N}$ gene of severe acute respiratory syndrome coronavirus 2(SARS-CoV-2). Virology. 549:1-4.

Yuasa, N., T. Taniguchi. and I. Yoshida. 1979. Isolation and some characteristics of an agent inducing anemia in chicks[J]. Avian. Diseases. 366-385 
Zhang, X., Y. Liu, B. Wu, B. Sun, F. Chen, J. Ji, J. Ma, and Q. Xie. 2013. Phylogenetic and molecular characterization of chicken anemia virus in southern China from 2011 to 2012. Sci. Rep. 3:3519.

Zhang, Y., N. Cui, N. Han, J. Wu, Z. Cui, and S. Su. 2017. Depression of Vaccinal Immunity to Marek's Disease by Infection with Chicken Infectious Anemia Virus. Front. Microbiol. 8:1863.

\section{Figures}
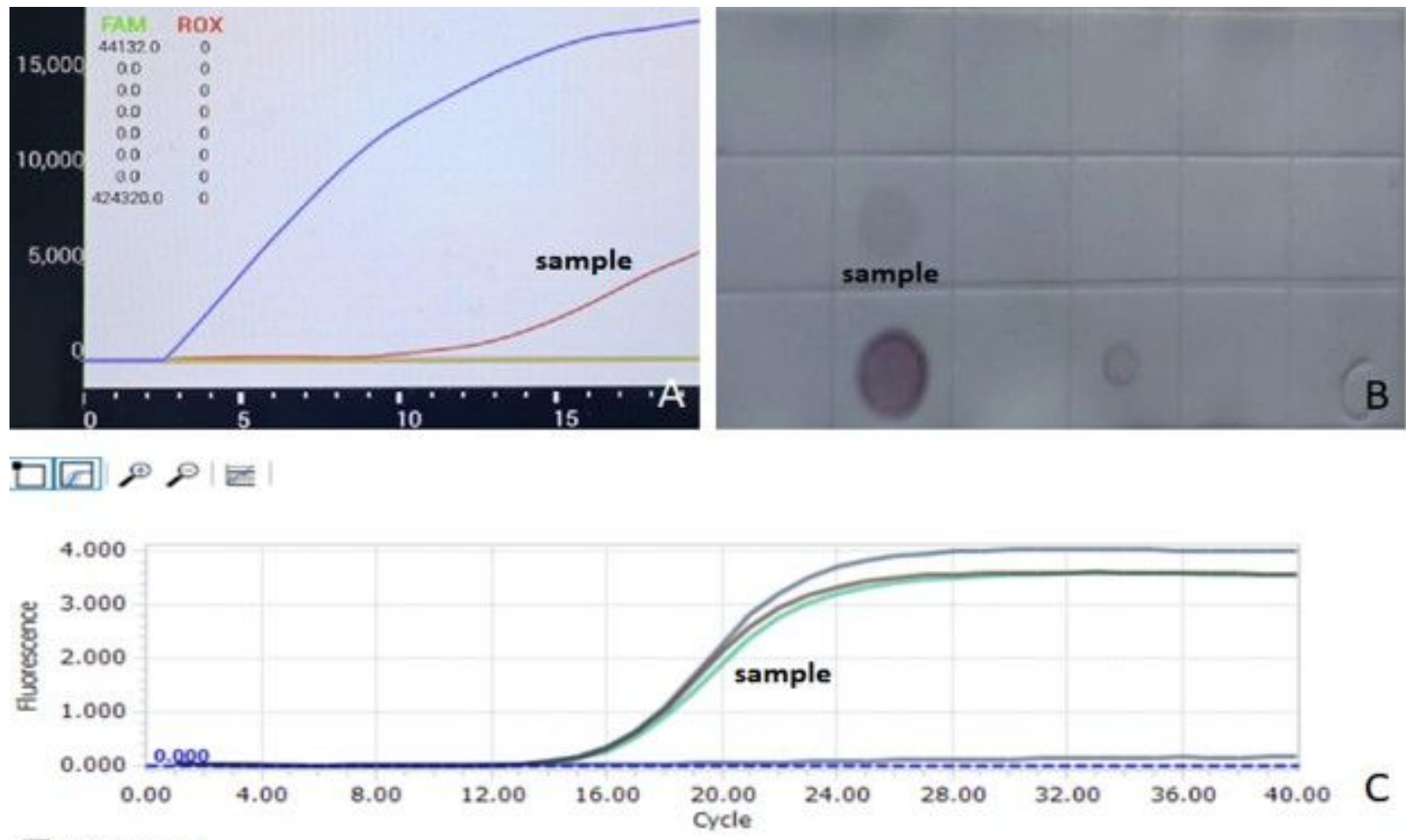

$\nabla$ SYBR Green I

\section{Figure 1}

Detection of CIAV nucleic acid in attenuated chicken pox vaccine RAA rapid assay (A), nucleic acid dot hybridization (B), and fluorescence quantitative PCR assay (C) were simultaneously adopted to detect CIAV contamination in vaccine samples, and all the methods showed positive results. 


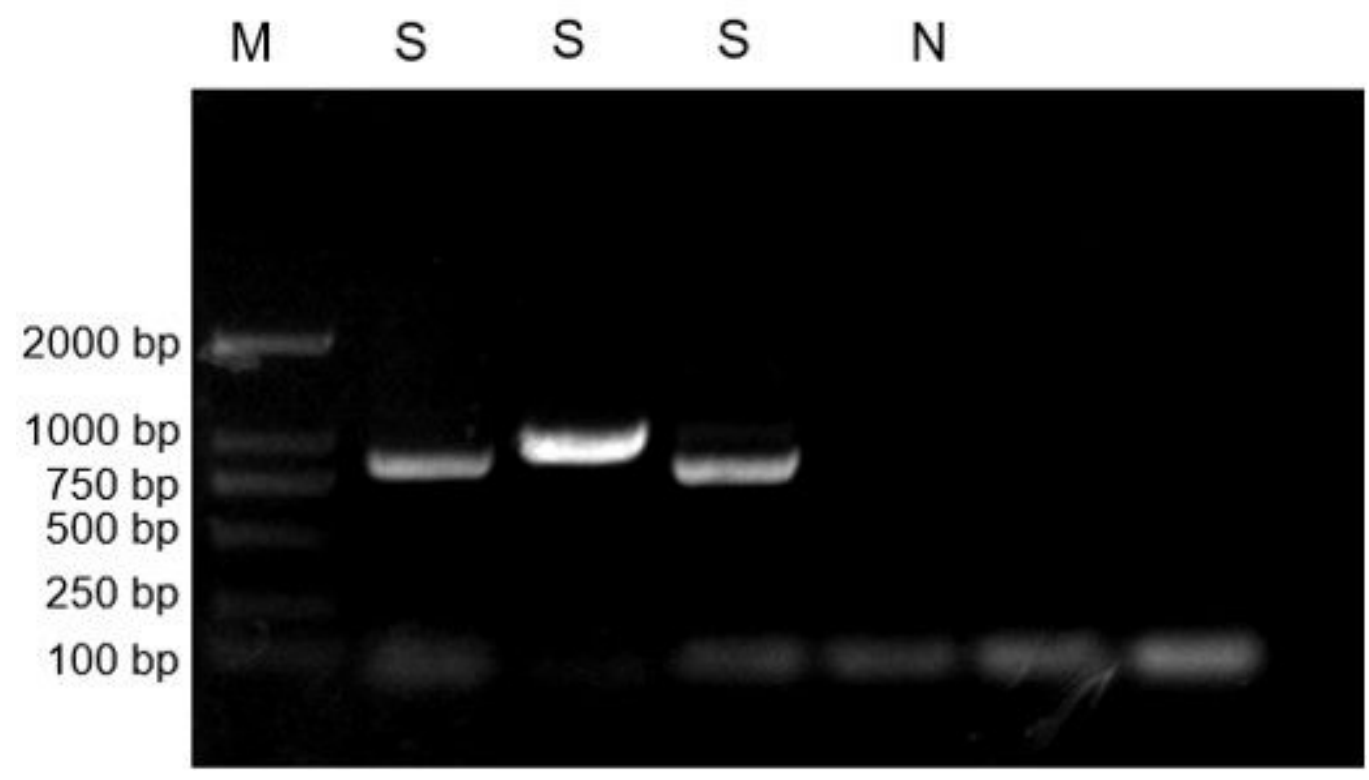

\section{Figure 2}

PCR amplication of CIAV in contaminated vaccine using three pairs of primers. M: marker; S: sample; N: negative

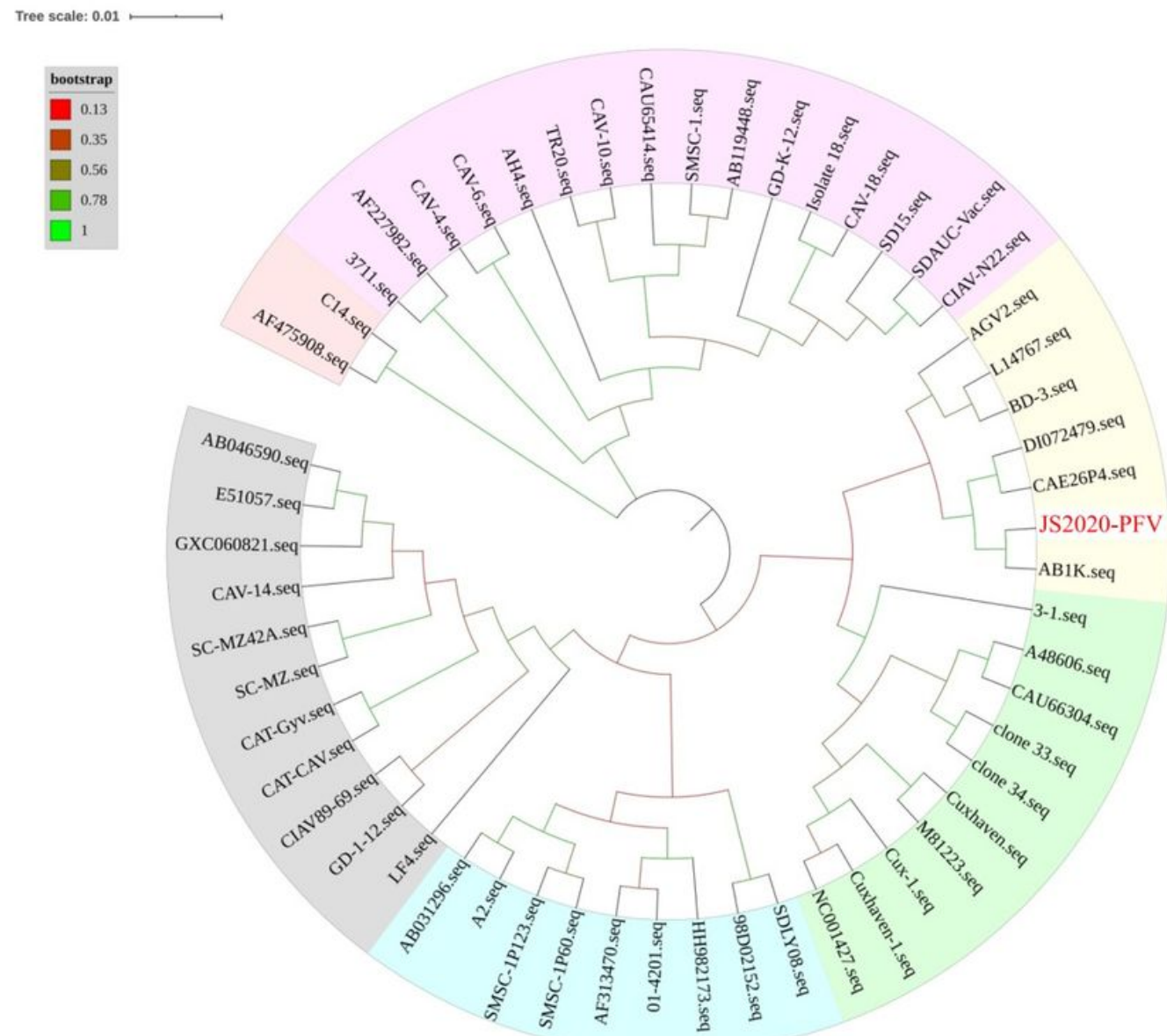


Figure 3

Phylogenetic tree of full-length viral genome of JS2020-PFV. The isolated strains have been highlighted in red The whole genome of the CIAV contaminant was then sequenced and named as JS2020-PFV (Genbank accession number: MW234428, 2296bp)

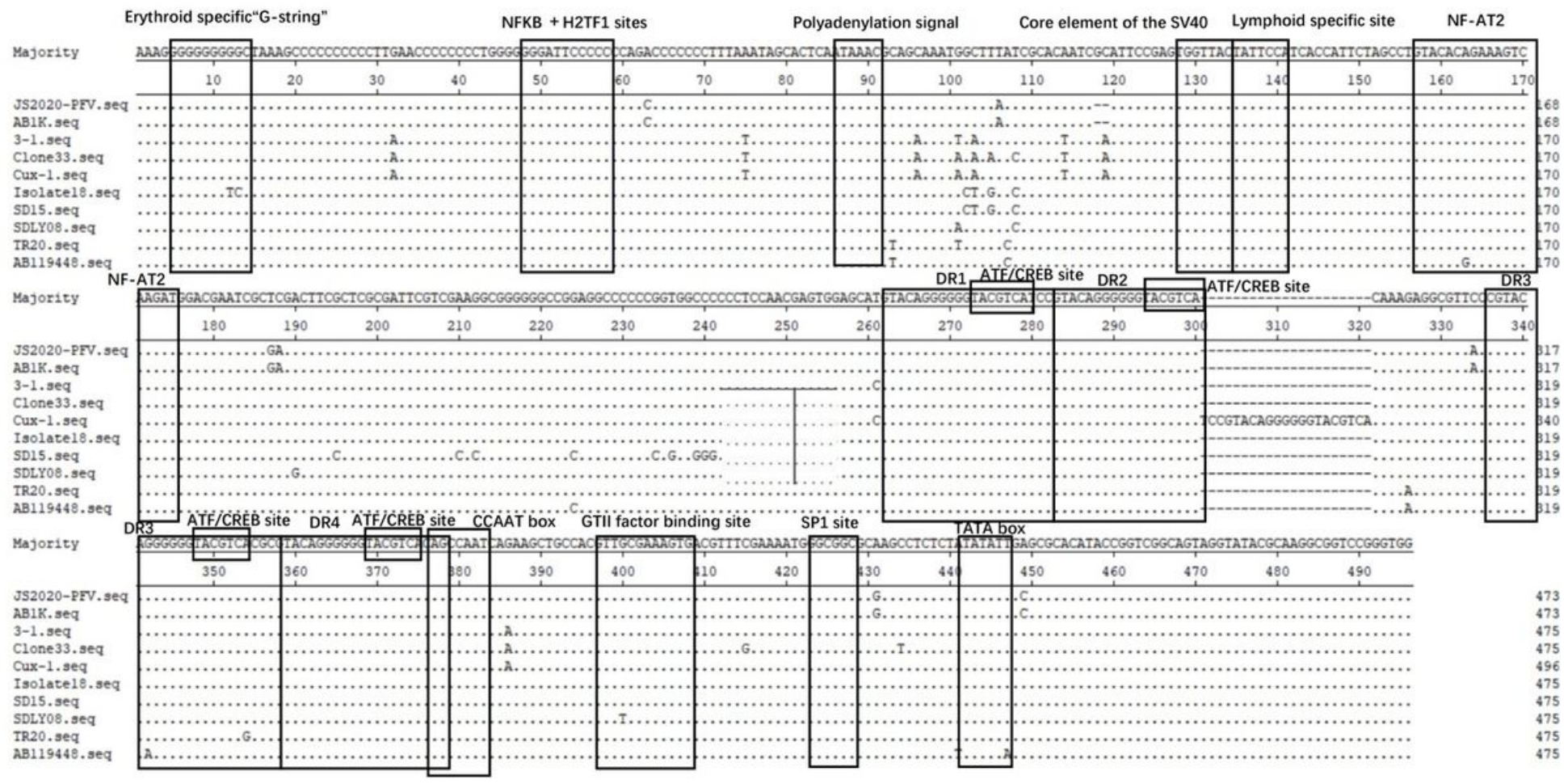

Figure 4

Structural analysis of the non-coding region of JS2020-PFV strain The sequences in black frames are the motifs of transcriptional regulatory elements in this study 